\title{
Terrain Feature Line Extraction by Improved Gradient-Based Profile Analysis
}

\author{
Kun Zou ${ }^{1}$ and Hongzhang Weng ${ }^{2}$ \\ ${ }^{1}$ School of Computer Engineering, University of Electronic Science and \\ Technology of China, Zhongshan Institute, Zhongshan, China \\ ${ }^{2}$ School of Computer Science and Engineering, University of Electronic Science \\ and Technology of China, Chengdu, China \\ cszoukun@foxmail.com
}

\begin{abstract}
The traditional terrain feature line extraction method using gradient-based profile analysis may induce feature discontinuity along flat ridges or valleys, and suffers a lot from terrain noises. To conquer this problem, an improved gradient-based profile analysis algorithm is proposed, and applied to the extraction of ridges and valleys from digital elevation models (DEMs). Firstly, the improved Sobel filter is adopted to capture the gradient direction, based on which the optimal profile direction is determined. Secondly, a global profile analysis process is devised to identify both strong and weak feature points. Then, features are extended along their feature directions, and final feature candidates are constructed by strong feature points and nearby weak ones with coinciding feature directions. After that, feature thinning, loop breaking, pruning and smoothing are conducted in succession to obtain final feature lines. Experiments are made by testing the proposed method and the traditional method on some real DEM data. It is observed that the proposed method performs much better in preserving feature continuity and resisting noises, and it can produce decent feature lines conforming to the terrain reality.
\end{abstract}

Keywords: Feature line extraction; Digital elevation model; Profile analysis; Terrain feature

\section{Introduction}

Ridge lines and valley lines delineate the most prominent skeleton structures of terrain. Their automatic extraction is of vital importance to topographic research, and can be applied to map-making, hazard prediction, geographical information systems [1] and sample-based terrain synthesis [2-3]. DEMs are easily available and ubiquitous in various applications. In consequence, terrain feature line extraction from DEMs is studied extensively.

So far, mainstream methods for terrain feature line extraction can be divided into two categories: water flow simulation-based methods [1,4-5] and geometrical morphological analysis-based methods [6-11]. The former conduct water flow simulation on terrain surfaces, in which course flow direction matrix and flow accumulation matrix are constructed, then valley lines are formed by concatenating feature points whose flow accumulation amount is above a predefined threshold, and ridge lines are obtained by extracting watershed boundaries. These methods can produce feature lines with great integrity, but often suffer from their inefficiency. Furthermore, the flow direction judgment on flat areas is usually a problem, and the output ridge line forms a closed loop, which does not match with the real case. 
Geometrical morphological analysis-based methods identify feature candidates by analyzing terrain profiles in local windows, and then concatenate feature points to extract terrain feature lines. Peucker and Douglas [6] propose a moving window method, which scans the DEM data with a $2 \times 2$ window, and labels the highest point in the window as ridge candidate and the lowest as valley candidate. Wood [7] determines whether a candidate is a ridge or valley point by examining extreme points of the second derivative. Chang et al. [8-9] put forward a method called PPA, which lowers the criteria for ridge candidates to have at least one lower neighbor on both sides, and conducts feature connection, polygon breaking, pruning and smoothing to accomplish feature line extraction. Zhang et al. [10] adapt PPA to improve the accuracy and speed of feature extraction. Huang and Liu [11] extract terrain feature lines by gradient-based profile analysis, which determines the approximate optimal profile direction based on the gradient orientation, and identifies the point with local maximum/minimum elevation as a ridge/valley point. Compared with water flow simulation-based methods, geometrical morphological analysis-based methods are usually much simpler and more efficient, and have higher feature accuracy. However, their results are easily influenced by the window size. In addition, these methods are sensitive to noises, and do not do well in preserving feature continuity. Although methods like PPA relieve the discontinuity problem by lowering the criteria for feature candidates, they pay the price with low efficiency in feature screening and low accuracy in feature location.

The traditional terrain feature line extraction method [11] using gradient-based profile analysis may induce feature discontinuity along flat ridges or valleys, and suffers a lot from terrain noises. This paper analyzes causes of these problems, based on which an improved gradient-based profile analysis algorithm is proposed, and applied to the extraction of ridge and valley lines from DEMs. By adopting the improved Sobel filter to capture the gradient direction, the optimal profile direction is determined more accurately. By using global profile analysis rather than analysis in local windows, feature candidates can be identified more correctly and less sensitively to noises, and the parameter tuning of window size is avoided. By conducting feature extension, feature continuity can be preserved much better. Furthermore, results with different feature strength can be obtained by setting different thresholds for identifying strong feature points. To obtain high quality feature lines, feature thinning, loop breaking, pruning and smoothing are applied in succession. The proposed method performs much better in preserving feature continuity and resisting noises, and it can produce decent feature lines conforming to the terrain reality.

\section{The Traditional Gradient-Based Profile Analysis Method}

The basic principle of feature line extraction based on profile analysis is to analyze profiles in DEM, find extreme points on profiles in terms of elevation, and take them as ridge or valley candidates. More specifically, maximum points are regarded as ridge candidates, while minimum points are regarded as valley candidates. Then, these candidates are filtered and sorted according to certain rules and required ridge or valley lines can be obtained.

Proper choice of profile direction is the assurance of obtaining reliable and correct results for profile analysis-based methods. The original method exploits only two orthogonal profiles (horizontal and vertical profiles), which may lose feature points on some ridges and valleys. Although this disadvantage can be overcome by adding a pair of profiles in diagonal directions, false feature points and computing load also increase with profiles.

Huang and Liu [11] point out that the essence of profile analysis-based methods is to determine watershed points and water collection points on local terrains, and it is based on the assumption that water flow direction of current analyzing point is perpendicular to 
profile direction, which usually does not agree with the fact. In theory, under the only action of gravity, a point on terrain surface has only one water flow direction, which is opposite to the direction of gradient. So they put forward a new method which selects the terrain profile perpendicular to the falling line (the opposite direction of gradient) to determine the candidate points on ridges and valleys, as described below.

Step 1. For each point in DEM, calculate its water flow direction as the opposite direction of gradient. The gradient vector $\left(f_{x}, f_{y}\right)$ is calculated as

$f_{x}=\frac{f(i+1, j)-f(i-1, j)}{2 \Delta_{x}}, f_{y}=\frac{f(i, j-1)-f(i, j+1)}{2 \Delta_{y}}$

in which $f(x, y)$ denotes the elevation of the point in DEM with coordinates $(x, y), \Delta_{x}$ and $\Delta_{y}$ indicate respectively the horizontal and vertical resolutions of the DEM, i.e., the distances between neighboring sampling points in corresponding directions.

Step 2. From the four main directions (with direction angles $0^{\circ}, 45^{\circ}, 90^{\circ}$, and $135^{\circ}$ ), choose the one approximately perpendicular to the water flow direction as the direction of terrain profile.

Step 3. Examine the center point of the selected terrain profile to see if it is an extreme point. When it is higher than neighboring points on both sides, it is a ridge point. And when it is lower than neighboring points on both sides, it is a valley point.

As a representative profile analysis-based method for terrain feature line extraction, the above algorithm makes a great improvement both in theory and performance. However, it has the following disadvantages.

1. As in (1), each component of the gradient vector is calculated with only one pair of points, which is easily affected by noises, and may lead to missing or wrong detection of some feature points.

2. The "higher than" and "lower than" criteria applied in a small local window are susceptible to noises and plausible features, which brings about lots of discontinuous short feature lines. In addition, flat features such as even-crested ridges cannot be properly handled, because a small detection window makes no difference between a plateau and a flat feature wider than itself.

3. There is no post-processing to increase the quality of the resulting feature lines, so results are not guaranteed to be one point wide and have plenty of unreal short branches.

To solve the above problems, we propose an improved method for terrain feature line extraction, as introduced in the next section.

\section{The Proposed Method}

The main procedures of the proposed method are shown in Figure 1. The input data can be any DEM with regular grids. At first, both strong and weak feature points are identified by feature detection. Then, during feature extension, both kinds of feature points are extended along their feature directions in which course the newfound feature points are also labeled as weak feature points, and feature candidates are constructed by strong feature points and nearby weak ones with coinciding feature directions. This is followed by polygon breaking, to break loops in the feature graph and make sure feature lines to be one point wide at the same time. At last, in pruning and smoothing, short branches are pruned and feature lines are smoothed to be more natural. The details of each procedure are given in the following. 


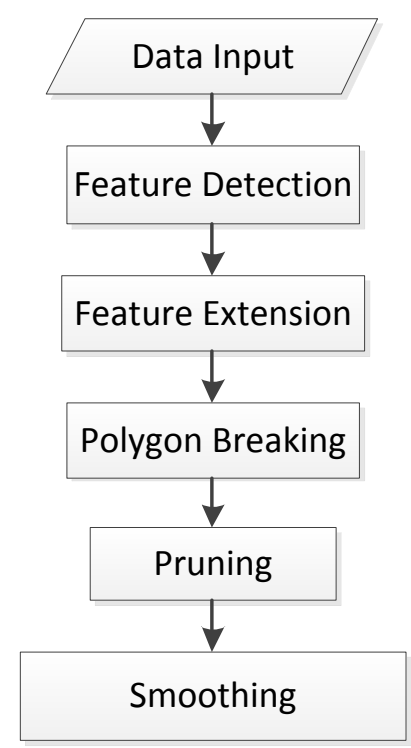

Figure 1. Flowchart of the Proposed Method

\subsection{Feature Detection}

Like the traditional gradient-based profile analysis method, our feature detection algorithm also selects the approximate optimal direction of terrain profile according to the water flow direction. However, we adopt the improved Sobel filter [12] to derive the gradient direction, and devise a global profile analysis process with two thresholds to identify both strong and weak feature points. The processing steps for each point $P_{i, j}$ in DEM are shown below.

Step 1. Calculate the water flow direction of $P_{i, j}$ by considering its eight neighbors in the DEM grid (shown in Figure 2). The improved Sobel filter can capture edge directions more accurately and are more robust to noises, the filter matrixes of which are

$$
H_{x}=\frac{1}{32}\left[\begin{array}{ccc}
-3 & 0 & 3 \\
-10 & 0 & 10 \\
-3 & 0 & 3
\end{array}\right], H_{y}=\frac{1}{32}\left[\begin{array}{ccc}
-3 & -10 & -3 \\
0 & 0 & 0 \\
3 & 10 & 3
\end{array}\right]
$$

So we compute the gradient vector $\left(f_{x}, f_{y}\right)$ by applying them to $P_{i, j}$ :

$f_{x}=\begin{aligned} & {[3 f(i+1, j-1)-3 f(i-1, j-1)+10 f(i+1, j)} \\ & -10 f(i-1, j)+3 f(i+1, j+1)-3 f(i-1, j-1)]\end{aligned} / 32 \Delta_{x}$

$f_{y}=\begin{aligned} & {[3 f(i+1, j-1)-3 f(i+1, j+1)+10 f(i, j-1)} \\ & -10 f(i, j+1)+3 f(i-1, j-1)-3 f(i-1, j+1)]\end{aligned} / 32 \Delta_{y}$

The symbols have the same meaning as in (1). When we calculate $f_{y}$ in (4), the coefficients of $H_{y}$ are inverted because we invert the $y$ axis as shown in Figure 3. 


\begin{tabular}{|c|c|c|}
\hline$P_{i-1, j-1}$ & $P_{i, j-1}$ & $P_{i+1, j-1}$ \\
\hline$P_{i-1, j}$ & $P_{i, j}$ & $P_{i+1, j}$ \\
\hline$P_{i-1, j+1}$ & $P_{i, j+1}$ & $P_{i+1, j+1}$ \\
\hline
\end{tabular}

Figure 2. Eight Neighbors of $\boldsymbol{P}_{i, j} \quad$ Figure 3. Water Flow Direction of $\boldsymbol{P}_{i, j}$

The water flow direction can be obtained by first computing the arc tangent:

$\theta_{0}=\arctan \left(\left(-f_{y}\right) /\left(-f_{x}\right)\right)$

in which $\theta_{0} \in\left(-90^{\circ}, 90^{\circ}\right)$. Then the angle $\theta$ between the water flow direction and $x$ axis (shown in Figure 3 ) which is in the interval $\left[0^{\circ}, 360^{\circ}\right]$ can be worked out as follows:

(1) If $f_{x}=0$ and $f_{y}=0, P_{i, j}$ is not a feature point, and there is no need to carry out Step 2 and Step 3.

(2) If $f_{x}=0$ and $f_{y}<0, \theta=90^{\circ}$.

(3) If $f_{x}=0$ and $f_{y}>0, \theta=270$.

(4) If $f_{x}<0$ and $f_{y}>0, \theta=360^{\circ}+\theta_{0}$.

(5) If $f_{x}>0, \theta=180^{\circ}+\theta_{0}$.

(6) In other cases, $\theta=\theta_{0}$.

Step 2. Select the direction of terrain profile which is approximately perpendicular to the water flow direction:

(1) If $\theta \leq 22.5^{\circ}, 157.5^{\circ}<\theta \leq 202.5^{\circ}$, or $\theta>337.5^{\circ}$, select the vertical terrain profile.

(2) If $22.5^{\circ}<\theta \leq 67.5^{\circ}$, or $202.5^{\circ}<\theta \leq 247.5^{\circ}$, select the $135^{\circ}$ terrain profile.

(3) If $67.5^{\circ}<\theta \leq 112.5^{\circ}$, or $247.5^{\circ}<\theta \leq 292.5^{\circ}$, select the horizontal terrain profile.

(4) If $112.5^{\circ}<\theta \leq 157.5^{\circ}$, or $292.5^{\circ}<\theta \leq 337.5^{\circ}$, select the $45^{\circ}$ terrain profile.

Step 3. Analyze the selected terrain profile to identify whether the current point $P_{i, j}$ is a strong feature point, a weak one or a non-feature point. Instead of using a small window, we devise a global profile analysis process. Figure 4 shows an example of it for ridge line extraction when the horizontal terrain profile is selected. Point-by-point scan is carried out along the selected profile in opposite directions from $P_{i, j}$ to measure the maximum elevation drops on both sides. Two thresholds $\left(T_{\text {high }}\right.$ and $\left.T_{\text {low }}\right)$ are predefined to identify strong and weak feature points respectively. $T_{\text {high }}$ is closely related to the feature saliency of the extraction result, while $T_{\text {low }}$ is used to filter out noises. In practice, there is no need to scan all the points. When the elevation drop is already larger than $T_{\text {high }}$ or a point higher than $P_{i, j}$ is found, scan in that direction can be stopped. The scan algorithm for direction $i$ $(i=1$ or 2$)$ is listed below, in which Flagi denotes which interval the detected maximum elevation drop locates in, and $f(P)$ means the elevation of $P$. 


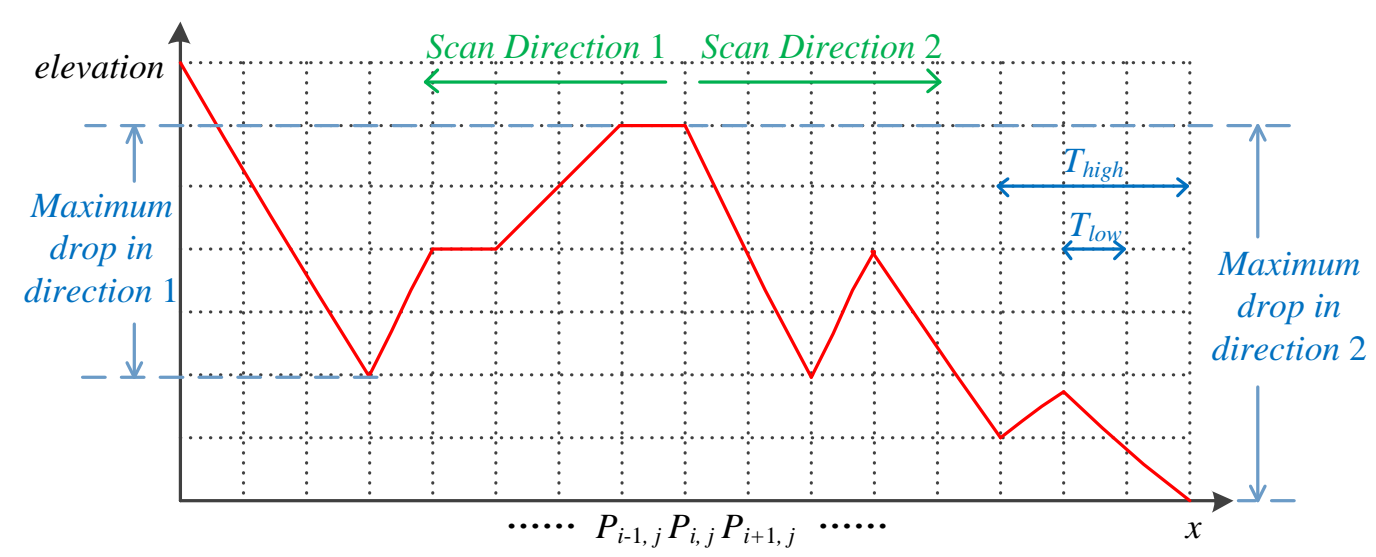

Figure 4. Illustration of the Global Profile Scan for $\boldsymbol{P}_{i, j}$

Step (1) Let Flagi $=0$.

Step (2) Take next point as $P_{c}$. If the profile boundary is reached or $f\left(P_{c}\right)>f\left(P_{i, j}\right)$, go to Step (4).

Step (3) Let $\Delta f=f\left(P_{i, j}\right)-f\left(P_{c}\right)$. If $T_{\text {low }}<\Delta f<T_{\text {high }}$, then Flagi $=1$, and go to $\operatorname{Step}(2)$; If $\Delta f>T_{\text {high }}$, then Flagi $=2$, and go to $\operatorname{Step}(4)$; If $\Delta f<T_{\text {low }}$, then go to $\operatorname{Step}(2)$.

Step (4) Return the value of Flagi.

Profile scan in two directions is conducted consecutively in this way to get Flag 1 and Flag2, and the identification result depends on Flag $=\min ($ Flag1, Flag2). There are three possibilities:

(1) Flag $=0$, then $P_{i, j}$ is not a feature point.

(2) Flag $=1$, then $P_{i, j}$ is a weak feature point.

(3) Flag $=2$, then $P_{i, j}$ is a strong feature point.

When the target is to extract valley lines, we measure the maximum elevation rises instead, and make corresponding changes of the above procedures.

Figure 5 gives an example of feature detection results for ridge line extraction. Figure 5 (a) shows the gray scale image of a DEM sample; Figure 5 (b) shows the detected strong feature points; Figure 5 (c) shows all detected ones. In Figure 5 (b) and (c), feature points with horizontal, vertical, $45^{\circ}$ and $135^{\circ}$ feature directions are shown in blue, red, yellow and green respectively.

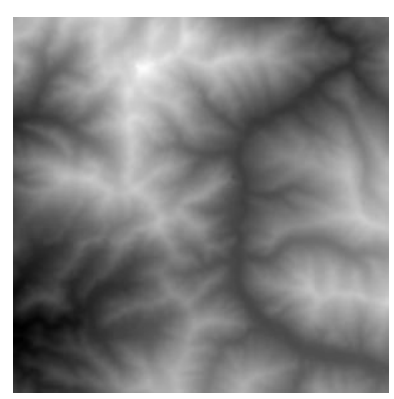

(a) A terrain sample



(b) Strong feature points

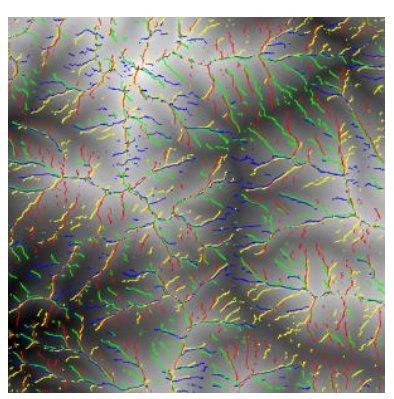

(c) All feature points

\section{Figure 5. Feature Detection Results for Ridge Line Extraction}

\subsection{Feature Extension}

After feature detection, we have got strong and weak feature points. As Figure 5 shows, feature lines constructed by concatenating all neighboring strong feature points can well exhibit main terrain structures, but are poor in continuity; even the feature lines composed of both kinds of feature points still have some discontinuities, and are too cluttered. We 
solve the problem by first conducting feature extension on all feature points to improve continuity, and then, in order to sweep away weak features and noises without compromising continuity, we search around strong feature points for weak ones (including the newly added feature points) with coinciding feature directions, and the final feature candidates are composed of strong feature points and the newfound ones.

\section{Feature extension}

For each feature point (no matter strong or weak), try to extend it along its feature direction, which is perpendicular to the direction of the selected terrain profile. Figure 6 gives an illustration, where feature extension for $P_{i, j}$ can be conducted along the upper left direction and down right direction respectively. Let's take the former as an example:

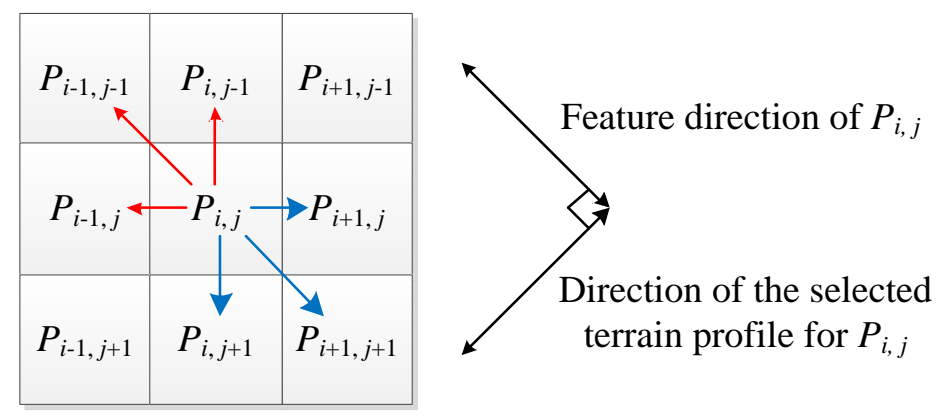

Figure 6. Illustration of Feature Extension for $\boldsymbol{P}_{i, j}$

(1) Examine $P_{i-1, j-1}, P_{i-1, j}$ and $P_{i, j-1}$, if any one of them is already a feature point, there is no need to go on feature extension along this direction, otherwise continue.

(2) For ridge line extraction: Let $P_{c}$ denote the highest point among $P_{i-1, j-1}, P_{i-1, j}$ and $P_{i, j-1}$. If $P_{c}$ is higher than its two neighboring points perpendicular to the direction from $P_{i, j}$ to $P_{c}$, label $P_{c}$ as a weak feature point and set its feature direction to be from $P_{i, j}$ to $P_{c}$. If the condition is not met, feature extension along this direction terminates. For valley line extraction, the aforementioned "highest" and "higher" should be substituted for "lowest" and "lower".

(3) Let $P_{c}$ take $P_{i, j}$ 's position, and try to conduct feature extension around it in the same way.

To avoid over-extension, a threshold is designated to limit the max extension length.

\section{Determination of feature candidates}

This step is similar to step 1. For each strong feature point, search for its neighboring weak ones (including the points newly labeled in step 1) along its feature direction. Let's take the same example in Figure 6, consumed that $P_{i, j}$ is a strong feature point, and we search for weak ones along the upper left direction:

(1) Examine $P_{i-1, j-1}, P_{i-1, j}$ and $P_{i, j-1}$, if any one of them is already a strong feature point, there is no need to go on searching along this direction, otherwise continue.

(2) Examine $P_{i-1, j-1}$, the higher of $P_{i-1, j}$ and $P_{i, j-1}$, and the lower in sequence (the sequence of the last two points should be exchanged for valley line extraction), and let $P_{c}$ denote the point to be examined. If $P_{c}$ is a weak feature point and the feature directions of $P_{c}$ and $P_{i, j}$ are the same or next to each other, label $P_{c}$ as a strong feature point. If none of the three points satisfies the criteria, searching along this direction terminates.

(3) Let $P_{c}$ take $P_{i, j}$ 's position, and repeat the above procedures.

In the end, all strong feature points constitute feature candidates.

Figure 7 shows the feature extension result for the DEM sample in Figure 6. Figure 7 (a) gives the result of step 1, where strong feature points, original weak ones, and newly added ones are shown in red, green and blue respectively. We can see that many isolated feature points or short feature lines are concatenated to more significant branches, and most of the gaps are filled up. Figure 7 (b) shows the result of step 2, in which the original 
strong feature points are shown in red and the newly labeled ones in blue. Apparently, this result is much less cluttered and has little sacrifice to continuity.

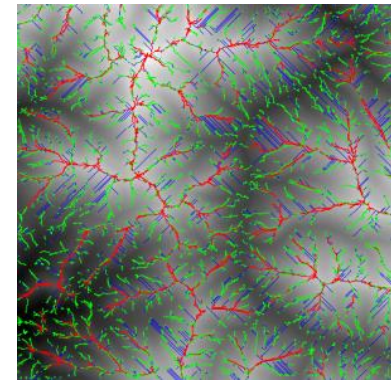

(a) Result of step 1

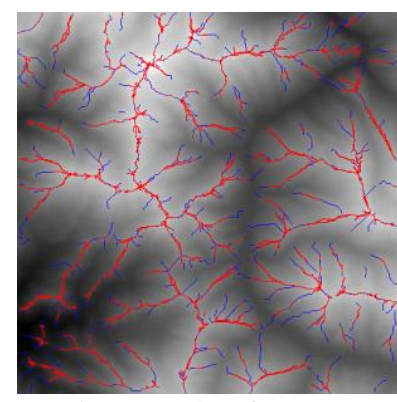

(b) Result of step 2

Figure 7. Feature Extension Result for Ridge Line Extraction

\subsection{Polygon Breaking}

After feature candidates are determined, feature graph is constructed, in which vertexes correspond to feature candidates and edges are formed by concatenating neighboring feature candidates. The weight of an edge is defined to be the sum of its two vertexes' height. When two edges are crossed, the edge with lower weight is eliminated. Figure 8 (a) shows the constructed graph.

There are some loops in the feature graph, and they should be broken. This procedure is called polygon breaking in [8], but this method is very slow. So we take the method in [3] which utilizes Kruskal minimum spanning tree algorithm to break loops, and Figure 8 (b) shows an example.

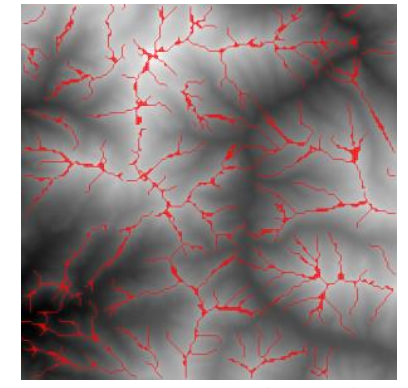

(a) Constructed graph



(b) Result of polygon breaking

Figure 8. Results of Graph Construction and Polygon Breaking

\subsection{Pruning and Smoothing}

There are many short branches in Figure 8 (b) which seldom exist in real terrains. So we take the method in [8] to prune short branches. Figure 9 (a) shows the result of pruning branches less than 5 edges.

Finally, feature lines are smoothed to be more natural with the method in [8], and Figure 9 (b) gives the result. 




(a) Result of pruning

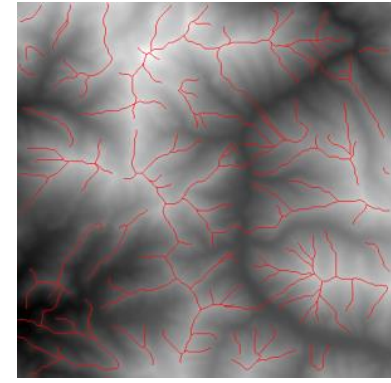

(b) Result of smoothing

Figure 9. Results of Pruning and Smoothing

\section{Experimental Results}

In order to verify the effectiveness of the proposed method, we make a comparison with the traditional gradient-based profile analysis method in [11]. The selected terrain samples are all $30 \mathrm{~m}$ grid DEMs of real terrain. During the implementation, $T_{\text {high }}$ and $T_{\text {low }}$ are set to 100 and 3 respectively, and both the maximum extension length and maximum branch length for pruning are set to 10 .

Figure 10 presents the experimental results. Figure 10 (a) is the grayscale image of the selected DEM sample. Figure 10 (b) shows the feature candidates identified by the method in [11]. In fact, it is also the final feature extraction result because there is no subsequent processing. As can be seen, there are many isolated short feature lines stemming from noises and fractures occur nearly everywhere. As a contrast, feature candidates obtained by our method are shown in Figure 10 (c), in which the original strong feature points are shown in red and feature points added by feature extension are shown in blue. Apparently, our result is much less cluttered and feature continuity is preserved much better. The robustness to noises is attributed to the two-threshold global profile analysis. And better performance at preserving feature continuity is largely due to our feature extension procedure, in addition, more accurate selection of profile direction and the global profile analysis also contribute to it. In order to further validate our improved gradient-based profile analysis method and make a more fair comparison, we adapt the method in [11] by adding the same post-processing procedures including graph construction, polygon breaking, pruning and smoothing. The ridge line extraction result of the adapted method is shown in Figure 10 (d), and our result is presented in Figure 10 (e). Compared with Figure 10 (b), discontinuities are more prominent in Figure 10 (d) as a result of pruning. While our result is much more closer to the real terrain ridges. Figure 10 (f) shows the valley lines extracted by our method, which also conforms to the reality on the whole.

As can be seen from Figure 10 (e) and (f), there are still a few fractures. This is because the selection of profile direction is only based on a local neighborhood, which is not guaranteed to be accurate. Increasing the neighborhood size may relieve this problem. 


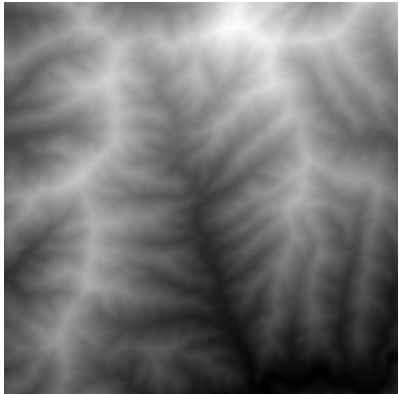

(a)

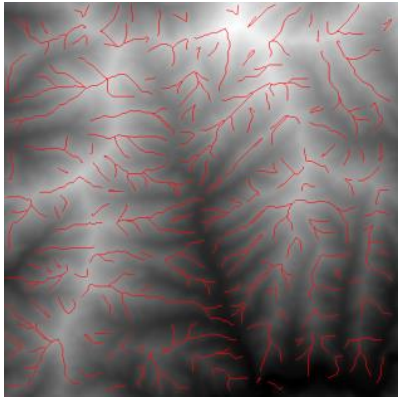

(d)

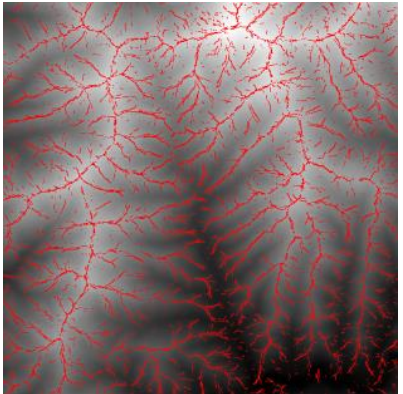

(b)

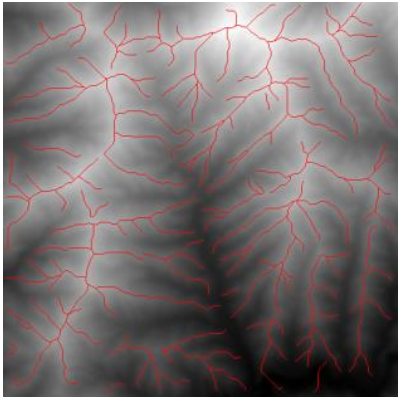

(e)

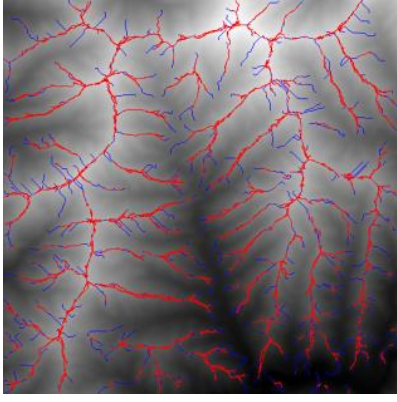

(c)

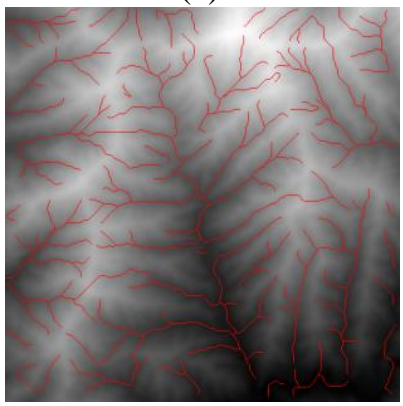

(f)

Figure 10. Experimental Results. (a) Grayscale image of the DEM sample; (b) Ridge candidates by [11]; (c) Our ridge candidates; (d) Ridge lines extracted by adapting the method in [11]; (e) Ridge lines extracted by our method; (f) Valley Lines Extracted by Our Method

Besides, some hook-like ends exist in the result, which can be eliminated by adding a hook remove procedure.

As another feature of the proposed method, results with different feature strength can be obtained by setting different thresholds for identifying strong feature points. Figure 11 gives an example, in which the ridge line extraction result with $T_{\text {high }}=100$ has much fewer ridge lines than the result with $T_{\text {high }}=10$, because many less prominent ridges are left out in the strong feature detection.



(a) DEM sample

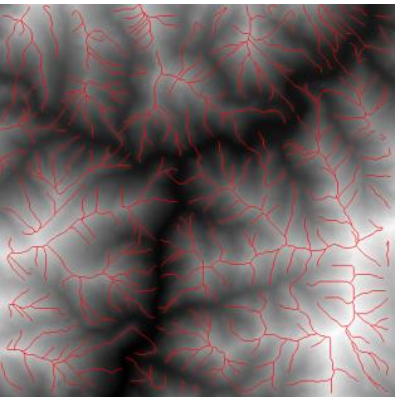

(b) $T_{\text {high }}=10$



(c) $T_{\text {high }}=100$

Figure 11. Ridge Line Extraction with Different $\boldsymbol{T}_{\text {high }}$

\section{Conclusions}

The traditional terrain feature line extraction method using gradient-based profile analysis is sensitive to noises and may induce feature discontinuity along flat ridges or valleys. To solve this problem, this paper proposes a two-threshold global profile analysis method and applies it to terrain feature line extraction. Compared with the traditional profile analysis in a small window, the global profile analysis is much 
more robust and can easily distinguish real feature points from noises and pseudo features, in which a low threshold is used to suppress noise and a high thre shold is used to control feature saliency of the extracted feature lines. In addition, a novel feature extension method is developed to obtain the final feature candidates, which makes a great contribution to better performance at preserving feature continuity. On the whole, the proposed method can produce decent feature lines that conform to the terrain reality. As future work, we would like to develop a more global method to select the profile direction more accurately.

\section{Acknowledgments}

This work is supported by the National Natural Science Foundation of China (Nos. 61502088, 61300095), the Natural Science Foundation of Guangdong Province (Nos. S2013010012307, S2012040011123), and the Cultivation Plan of the Outstanding Young Teachers in Guangdong Province (Nos. Yq2013206, Yq2015241, Yq2015242).

\section{References}

[1] A. Tribe, "Automated Recognition of Valley Lines and Drainage Networks from Grid Digital Elevation Models: A Review and A New Method”, Journal of Hydrology, vol. 139, no.1-4, (1992), pp. 263-293.

[2] H. Zhou, J. Sun and G. Turk, "Terrain Synthesis from Digital Elevation Models", IEEE Transactions on Visualization and Computer Graphics, vol. 13, no. 4, (2007), pp. 834-848.

[3] F. P. Tasse, J. Gain and P. Marais, "Enhanced Texture-Based Terrain Synthesis on Graphics Hardware", Computer Graphics Forum, vol. 31, no. 6, (2012), pp. 1959-1972.

[4] W. Guo, S. Liu, P. Yu and J. Xu, "Automatic Extraction of Ridgelines Using Drainage Boundaries and Aspect Difference”, Science of Surveying and Mapping, vol. 36, no. 6, (2011), pp. 210-212, 191.

[5] R. Barnes, C. Lehman and D. Mulla, "An Efficient Assignment of Drainage Direction over Flat Surfaces in Raster Digital Elevation Models", Computers and Geosciences, vol. 62, (2014), pp. 128-135.

[6] T. K. Peucker and D. H. Douglas, "Detection of Surface-Specific Points by Local Parallel Processing of Discrete Terrain Elevation Data", Computer Graphics and Image Processing, vol. 4, no. 4, (1975), pp. 375-387.

[7] J. Wood, "The Geomorphological Characterization of Digital Elevation Models", Leicester, UK: University of Leicester, (1996).

[8] Y. C. Chang, G. S. Song and S. K. Hsu, "Automatic Extraction of Ridge and Valley Axes Using the Profile Recognition and Polygon-Breaking Algorithm", Computers \& Geosciences, vol. 24, no. 1, (1998), pp. 83-93.

[9] Y. C. Chang and G. Sinha, "A Visual Basic Program for Ridge Axis Picking on DEM Data Using the Profile-Recognition and Polygon-Breaking Algorithm", Computers \& Geosciences, vol. 33, no. 2, (2007), pp. 229-237.

[10] H. Zhang, Y. Liu, Z. Ma, X. He and N. Bao, "A Terrain Skeleton Feature Extraction Method Based on Morphological Encoding”, Journal of Computer Research and Development, vol. 52, no. 6, (2015), pp. 1409-1423.

[11] P. Huang and Z. Liu, "Extraction of Ridge and Valley from DEM Based on Gradient", Geomatics and Information Science of Wuhan University, vol. 30, no. 5, (2005), pp. 396-399.

[12] B. Jähne, Editor, "Digitale Bildverarbeitung”, Springer-Verlag, Berlin, fifth edition, (2002).

\section{Authors}

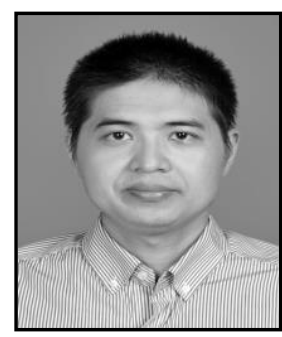

Kun Zou, he received his $\mathrm{PhD}$ degree in Computer Application Technology from South China University of Technology. He is currently an associate professor at University of Electronic Science and Technology of China, Zhongshan Institute. His research interests include terrain feature extraction, terrain synthesis and texture synthesis. 


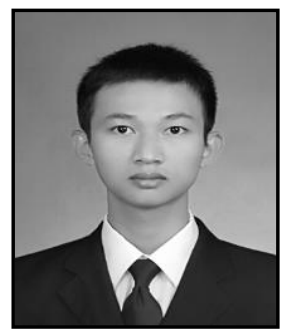

Hongzhang Weng, he received his bachelor's degree in Computer Science and Technology from Chongqing University of Posts and Telecommunications. He is currently a master student of Computer Application at University of Electronic Science and Technology of China. His research interests include terrain feature extraction and terrain synthesis. 